Rabaska

Revue d'ethnologie de l'Amérique française

\title{
Laboratoire de muséologie et d'ingénierie de la culture (LAMIC)
}

\section{Philippe Dubé}

Volume 3, 2005

URI : https://id.erudit.org/iderudit/201746ar

DOI : https://doi.org/10.7202/201746ar

Aller au sommaire du numéro

Éditeur(s)

Société québécoise d'ethnologie

ISSN

1703-7433 (imprimé)

1916-7350 (numérique)

Découvrir la revue

Citer ce document

Dubé, P. (2005). Laboratoire de muséologie et d'ingénierie de la culture

(LAMIC). Rabaska, 3, 210-213. https://doi.org/10.7202/201746ar d'utilisation que vous pouvez consulter en ligne.

https://apropos.erudit.org/fr/usagers/politique-dutilisation/ 


\section{Laboratoire de muséologie et d'ingénierie de la culture (LAMIC)}

Pavillon Charles-De Koninck, bureau 4291 Téléphone : (418) 656-5130 Université Laval ou (418) 656-2131, poste 3296 Québec (Québec) G1K 7P4 Courriel : philippe.dube@hst.ulaval.ca franccote@yahoo.ca

\section{Présentation}

Le Laboratoire de muséologie et d'ingénierie de la culture (LAMIC) deviendra au cours des prochains mois le premier centre canadien de muséologie expérimentale, l'un des rares du genre dans le monde. Actif comme regroupement de chercheurs depuis mars 2004, son infrastructure, financée dans le cadre du Fonds canadien d'innovation, se concrétisera à la fin de l'automne 2005.

Le LAMIC entend étudier les modalités de transmission de la culture à travers l'expérience muséale. Il s'attarde particulièrement à vérifier la réelle efficacité des technologies de pointe à transmettre des contenus culturels (connaissances, valeurs, repères identitaires). Ses recherches sur l'objet, l'espace et le visiteur, thématiques abordées à la fois sous l'angle du réel (expérimentation) et du virtuel (simulation), bénéficient de l'expertise et des réseaux d'une équipe de chercheurs chevronnés provenant de domaines aussi 
diversifiés que l'archéologie, l'architecture, les arts électroniques, le design industriel, l'ethnologie, le génie électrique et informatique, la géographie, l'histoire, la muséologie, la robotique, la scénographie, la sociologie et la tracéologie. Le LAMIC agit essentiellement en tant que plate-forme de collaboration entre divers groupes de recherche existants et comme centre de transfert technologique pour le milieu des musées et des firmes muséographiques. Il répond aux besoins urgents d'adaptation des musées face à l'émergence de nouvelles technologies de l'information et des communications, ainsi qu'à la nécessité d'une formation universitaire supérieure liée aux nouveaux territoires de recherche qui en découlent. Les musées canadiens et québécois se trouvent en effet au seuil d'un important changement de paradigme technologique. Pour y faire face, le milieu professionnel de la muséologie a besoin de nouveaux repères. C'est ce qu'entend apporter notre programme de recherche.

Profitant de l'attribution du plus imposant espace de recherche disponible sur le campus de l'Université Laval (premier niveau de l'ex-Centre muséographique, au pavillon Louis-Jacques-Casault), le LAMIC rejoint le Laboratoire des nouvelles technologies de l'image, du son et de la scène (LANTISs) et fait de la conjugaison de deux laboratoires un complexe de recherche en sciences de la culture. Occupant 905 mètres carrés d'une infrastructure muséographique de grande qualité, il la double d'équipements de recherche de haut niveau technologique, dont notamment le numérisateur de captation 3D le plus performant de l'est du Canada. Le LAmIC y accueillera un carrefour de collaborations entre des chercheurs et des partenaires extérieurs, un lieu synergique nourri par la rencontre de réseaux de recherche solidement établis. Au nombre des partenaires principaux, on compte d'abord le Musée de la civilisation du Québec, le Musée d'art contemporain de Montréal et la Pulperie de Chicoutimi, trois institutions où le LAMIC dispose d'antennes (espaces de travail et partenariats de recherche). La Société des arts technologiques (SAT, Montréal), MCG3D, É-Motion Solutions, L'Institut de technologie de l'information du Conseil national de recherches du Canada (ITI - CNRC), et la Chaire Fernand-Dumont sur la culture (INRS-Urbanisme, culture et société) sont également étroitement associés au laboratoire. Le visiteur-expert, quant à lui, occupe une place prépondérante au sein de la dynamique de recherche et ce, à titre de premier spécialiste apte à juger de l'efficacité des méthodes et instrumentations de transmission de la culture mises sous examen.

\section{Problématique de recherche}

La question de la transmission de la culture dans le musée demeure encore entière, car elle n'a jamais été abordée de manière globale, encore moins de 
façon systématique. Cependant, on a beaucoup étudié les visiteurs de musée (les publics et les non-publics de musée), notamment du point de vue de leur composition sociale et du type de visites qu'ils font, selon plusieurs critères, à savoir : âge, classe sociale, scolarité, activité de loisir culturel, tourisme culturel, périodicité des fréquentations, etc. On a aussi approfondi le champ de la réception de l'offre culturelle dans les musées à partir de méthodes liées à l'évaluation cognitive, à la communication scientifique ou encore d'autres approches propres à l'éducation dite muséale. On a de plus développé des problématiques qui tentaient de cerner les diverses dimensions de l'expérience de visite du musée en tenant pour acquis qu'il y avait bel et bien transmission de la culture ou, à tout le moins, transfert de connaissances sinon d'informations - relatives aux thématiques présentées sous forme d'expositions ou d'activités dans le musée. Les approches privilégiées pour l'ensemble de ces études sont à la fois d'ordres quantitatif (typologie des visiteurs et des fréquentations) et qualitatif (réception des présentations), couvrant ainsi des préoccupations de nature sociologique, historique, anthropologique, politique, linguistique, économique, communicationnelle ou encore éducative. De plus, quelques études ont abordé des questions de nature muséographique en explorant la dimension architectonique du musée à travers les espaces organisés à ses fins. Mais encore là, c'est la perception du visiteur qui retient surtout l'attention. Voilà donc un domaine qui nous a instruit - depuis une bonne vingtaine d'années de façon soutenue - sur l'expérience du visiteur de musée et plus globalement, sur les bénéfices qu'il en retire, selon de nombreux contextes qui apportent d'aussi nombreuses variables.

Mais quand il s'agit d'aborder la question holistique de la transmission de la culture dans le musée et tenter d'en dévoiler le processus à travers l'énonciation de ses modalités, nous avons affaire à un terrain plutôt vierge, pour ainsi dire intouché. On a toujours présumé qu'il y avait transmission sans en vérifier le procédé. Il est vrai que l'étude de cette dimension exige l'examen de quelques préalables qu'il faudra, dans un premier temps, éclairer. On devra d'abord spécifier ce que l'on entend par culture. Puis, voir comment s'articule et s'opère la transmission à partir de ce que nous en connaissons. Et enfin, approfondir le contexte muséal comme lieu d'accueil public qui possède un caractère et des particularités propres en regard de la transmission de la culture.

Le point de départ de cette interrogation trouve son origine dans une critique radicale de la muséologie comme théorie du musée qui nous semblait aller nulle part sur le plan épistémologique (théorie de la connaissance). De notre point de vue, la muséologie se trouvait donc dans une impasse théorique où la connaissance sur le musée empêchait jusqu'à certain point la réflexion 
sur lui-même parce qu'elle n'était pas - cette théorie - inspirée par plus grand qu'elle-même. Nous étions donc pris au piège, cantonné dans un culde-sac qui rendait tout dépassement impossible et pour en sortir, il fallait faire chemin arrière et revenir en bon radical à la racine, au fondement même du musée, soit en amont de ses fonctions (conserver - étudier - diffuser) en refondant la raison d'être du musée qui est celle avant tout de transmettre. $\mathrm{Ce}$ chemin à rebours n'aura pas été facile. Nous nous perdions littéralement dans les dédales labyrinthiques de définitions qui décrivent le musée plus qu'elles ne l'expliquent. Au bout du compte, nous avons été amené à penser que la transmission de la culture est le fondement ontologique du musée, celui qui peut non seulement nous permettre de le comprendre mais aussi de le situer parmi les autres acteurs de la culture dans la société. Nous sommes donc conscients des difficultés sémantiques, voire même idéologiques, d'une telle proposition mais il nous a semblé que ce nouvel angle de regard nous permettait de sortir des ornières qui, par définition, limitent la vue et surtout le point de vue que nous recherchions. Nous ne sommes évidemment pas en train de suggérer en douce que nous possédons la vérité sur le musée, mais admettons que nous sommes maintenant plus à l'aise pour entrevoir un horizon pour lui, et du coup pour nous.

\section{Projets}

Le LAMIC est actuellement investi dans plus d'une dizaine de projets de recherche. Parmi ceux-ci on compte : la mise en place d'un centre de numérisation 3D d'artefacts à l'Université Laval, l'étude de la conservation préventive d'œuvres hybrides en arts technologiques, l'incubation d'une entreprise de « réalité augmentée », la coréalisation d'expositions (Musée de l'Homme de Paris, Biennale de Venise 2005, Musée d'art contemporain de Montréal), l'application de la numérisation 3D au processus de fouille archéologique, et le développement de technologies de télévisite et d'analyse de sites historiques via la toile électronique.

Les équipements du LAMIC seront accessibles dès 2006 à tous les chercheurs, créateurs et entreprises intéressés à explorer les modalités de transmission de la culture en contexte muséal. 